\title{
High order accurate numerical simulation of flow past an oscillating circular cylinder
}

\section{Introduction}

Over the years, flow past circular cylinder has been studied ${ }^{1-11}$ extensively due to its academic and practical significance. Periodic lift and drag forces are generated over the cylinder due to separation and vortex shedding leading to structural vibration also known as vortex induced vibrations (VIV). This fluid-structure interaction (FSI) may result in serious engineering problems such as fatigue failure of the off-shore riser and sub-sea pipelines. Therefore, prediction of fluid forces on the cylinder has great importance from the point of view of structural design. Often, two types of FSI problems involving cylinder are studied. In one case, cylinder oscillates due to the in-line and transverse forces generated by vortex shedding. ${ }^{1-10}$ In the second case, the cylinder is forced to oscillate in a transverse direction with prescribed oscillating amplitude and excitation frequency, known as force induced vibration (FIV). ${ }^{11}$ In this paper, the FSI problems are studied using a numerical method. An accurate Harten Lax and van Leer with contact for artificial compressibility (HLLC-AC) Riemann solver ${ }^{12-14}$ developed for solving incompressible flows in artificial compressibility formulation have been used for flow computation. The Riemann solver is modified to incorporate Arbitrarily Lagrangian-Eulerian (ALE) ${ }^{15}$ formulation in order to take care of mesh movement in the computation, where radial basis function ${ }^{16}$ is used for dynamically moving the mesh. Higher order accuracy is achieved using quadratic solution reconstruction based on solution dependent weighted least squares (SDWLS) ${ }^{17}$ The results obtained by the present method is validated those reported in the literature. ${ }^{1-11}$

\section{Arbitrarily Lagrangian-Eulerian (ALE) ${ }^{15}$ formulation}

An artificial compressibility ${ }^{18}$ based, with dual-time stepping, the unsteady Navier-Stokes incompressible equations is modified here to take care of moving boundaries using the Arbitrarily LagrangianEulerian (ALE) $)^{15}$ formulation. The integral form of the twodimensional governing equation in arbitrarily Lagrangian-Eulerian form can be written as

$\iint_{\Omega} \frac{\partial \mathrm{W}}{\partial \tau} \mathrm{dxdy}+\mathrm{I}^{\mathrm{M}} \iint_{\Omega} \frac{\partial \mathrm{W}}{\partial \mathrm{t}} \mathrm{dxdy}+\Theta^{\mathrm{M}} \oint\left[\left(\mathrm{E}^{\mathrm{c}}+\mathrm{E}^{\mathrm{v}}\right) \mathrm{n}_{\mathrm{x}}+\left(\mathrm{F}^{\mathrm{c}}+\mathrm{F}^{\mathrm{v}}\right) \mathrm{n} \mathrm{y}\right] \mathrm{dA}=\iint_{\Omega} \mathrm{S}_{0} \mathrm{dxdy}$
Volume 2 Issue 5 - 2018

Sonawane CR, More YB

Department of Mechanical Engineering, Symbiosis Institute of Technology(SIT), India

Correspondence: Sonawane CR, Department of Mechanical Engineering, Symbiosis Institute of Technology(SIT), Pune, India, Email crsonavane@gmail.com

Received: October 25, 2018 | Published: November 29, 2018

$$
\begin{aligned}
& W=\left\{\begin{array}{c}
p \\
\rho \\
u \\
v
\end{array}\right\} ; E^{c}=\left\{\begin{array}{c}
U \\
U u+p / \rho \\
U v
\end{array}\right\} ; G^{c}=\left\{\begin{array}{c}
V \\
u V \\
v V+p / \rho
\end{array}\right\} ; \\
& E^{v}=\left\{\begin{array}{c}
0 \\
\sigma_{x x} \\
\sigma_{x y}
\end{array}\right\} ; G^{v}=\left\{\begin{array}{c}
0 \\
\sigma_{y x} \\
\sigma_{y y}
\end{array}\right\} \\
& I^{M}=\left\{\begin{array}{lll}
1 & 0 & 0 \\
0 & 1 & 0 \\
0 & 0 & 1
\end{array}\right\} ; \quad \Theta^{M}=\left\{\begin{array}{lll}
\beta^{2} & 0 & 0 \\
0 & 1 & 0 \\
0 & 0 & 1
\end{array}\right\} ; S_{0}=\left\{\begin{array}{c}
0 \\
f_{e, x} \\
f_{e, y}
\end{array}\right\}
\end{aligned}
$$

Where $U=u-x_{t}$ and $V=v-y_{t}$ are the convective velocities in referential frame with $x_{t}$ and $y_{t}$ are the velocities of the moving grid in $\mathrm{X}$ and $\mathrm{Y}$ directions respectively. Note that, equation (1) does not exhibit any physical meaning until pseudo time steady state, i.e. $\left(\frac{\partial \mathrm{p}}{\partial \tau}=\frac{\partial \mathrm{u}}{\partial \tau}=\frac{\partial \mathrm{v}}{\partial \tau} \cong 0\right)$ is reached. As the pseudo-steady state is reached, the equations are identical to the original unsteady incompressible Navier-Stokes equations in ALE form. Now splitting the convective fluxes $\left(E^{c}\right.$ and $G^{c}$ ) of equation (1) into stationary reference flux and ale flux part as,

$$
\iint_{\Omega} \frac{\partial \mathrm{W}}{\partial \hat{\mathrm{o}}} \mathrm{dxdy}+I^{M} \iint_{\Omega} \frac{\partial \mathrm{W}}{\partial \mathrm{t}} \mathrm{dxdy}+\Theta^{M} \oint_{A}\left[\left(\mathrm{E}_{\mathrm{St}}^{\mathrm{c}}+\mathrm{E}^{\mathrm{v}}\right) \mathrm{n}_{\mathrm{x}}+\left(\mathrm{F}_{\mathrm{st}}^{\mathrm{c}}+\mathrm{F}^{\mathrm{v}}\right) \mathrm{n}_{\mathrm{y}}\right] d A-\oint_{A}\left[\left(\mathrm{E}_{\text {ale }}^{\mathrm{c}}\right) \mathrm{n}_{\mathrm{x}}+\left(\mathrm{F}_{\text {ale }}^{\mathrm{c}}\right) \mathrm{n}_{\mathrm{y}}\right] d A=\iint_{\Omega} \mathrm{S}_{0} \mathrm{dxdy}
$$

Now equation (4) can be discretized in a very similar manner to that for unsteady Navier-Stokes equation for stationary boundary problem. ${ }^{12-14}$ The additional effort need to be added for ale flux vector. This additional term, ale flux, is nothing but the volumetric increment along the face and can be evaluated by considering the Geometric Conservations Law (GCL). ${ }^{19}$ The radial basis function: ${ }^{16}$ Thin-Plate Spline (TPS) with global support is used for mesh movement. The Thin Plate Spline with global support generates meshes of high quality after deformation along with the computational efficiency. The fluxes at cell interface, that is, the stationary reference convective fluxes are evaluated using the Harten Lax and van Leer with contact for artificial compressibility (HLLC-AC) ${ }^{12-14}$ Riemann solver where interface values are reconstructed based on solution dependent weighted least squares (SDWLS). ${ }^{17}$ In the present case, Higher order accuracy is achieved using quadratic solution reconstruction. For viscous fluxes, a central differencing method based on Green-Gauss approach is used. 


\section{Results and discussion}

In the present paper, following two different cases for an oscillating circular cylinder is simulated. Figure 1 shows the domain considered as well as boundary conditions applied. Based on the mesh convergence study, a quad grid having 13840 mesh element and 13620 nodes is selected for the simulation. Case (1) Vortex-induced vibration (VIV) at Reynolds number $(\mathrm{Re})$ 150: Here, an equation of motion is used to represent VIV of a cylinder oscillating in the transverse direction (normal to the flow) as: $\mathrm{m} \ddot{\mathrm{y}}+\mathrm{c} \dot{\mathrm{y}}+\mathrm{ky}=\mathrm{F}$ where, $\mathrm{m}=$ structural mass, $\mathrm{c}=$ structural damping coefficient, $\mathrm{k}=$ spring constant, and $\mathrm{F}$ = fluid force acting in the transverse direction (lift force). Figure 2 shows the comparison of the results obtained with the literature ${ }^{7,8,10}$ against the displacement amplitude of the cylinder which is free to vibrate in the transverse direction for various reduced velocities $\left(\mathrm{U}_{\mathrm{r}}=\mathrm{U} / \mathrm{Df}_{\mathrm{n}}\right)$ at $\mathrm{Re}=150$. From Figure 2, it can be seen that the simulation results produced using present formulation agree well with the literature data. ${ }^{7,810}$ The maximum transverse amplitude occurs at a reduced velocity $U_{r}=4$. with $\mathrm{Y}_{\max }=0.5316$. Hence we can infer that the lock-in region for $\mathrm{Re}=150, \mathrm{~m}=8 / \pi$ lies at $U_{r}=4$. Case (2) Forced induced vibrations (FIV) at Reynolds number (Re) 100: simulation of the forcefully oscillating cylinder having diameter $\mathrm{D}=1$ is performed here. The transverse motion, $y(t)$ is given by the harmonic oscillation equation: $y(t)=A \sin \left(2 \pi f_{0} t\right)$ where, maximum amplitude, $A=0.25$ with oscillation frequency, $f_{0}=0.084$ is used, which is similar to that of literature. ${ }^{11}$ Figure 3 shows the comparison of the results obtained with the literature data ${ }^{11}$ against the Lift co-efficient and time. It can be seen that the results agree well with the results of Placzek et al. ${ }^{11}$

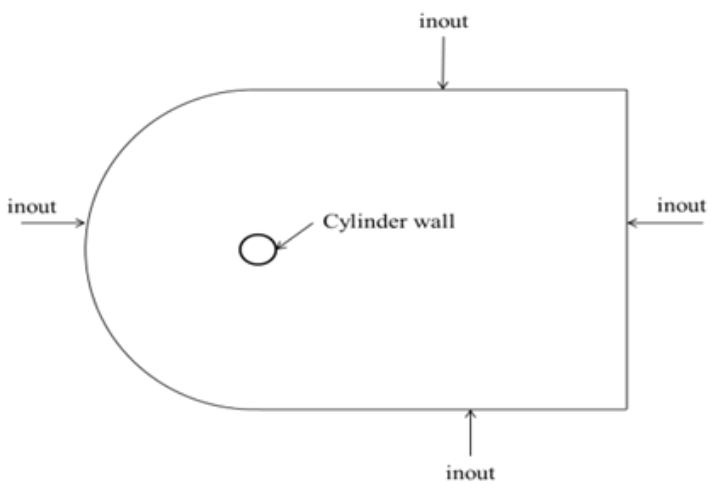

A Domain used

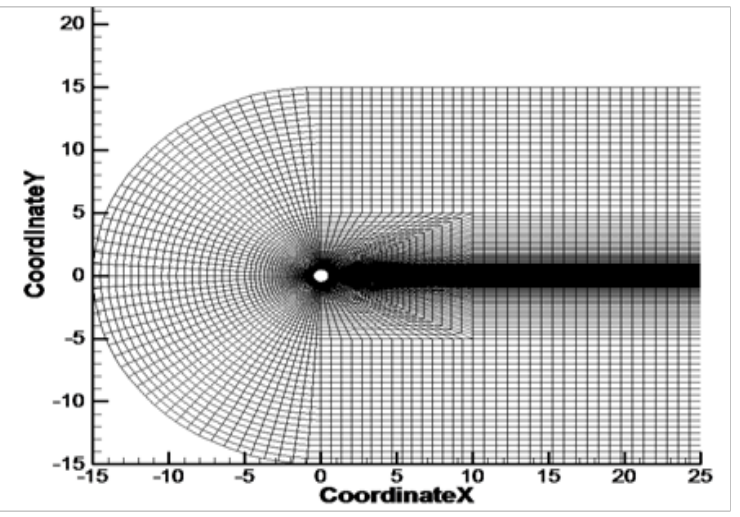

B Mesh used

Figure I Domain and mesh used for an Oscillating circular cylinder problem.

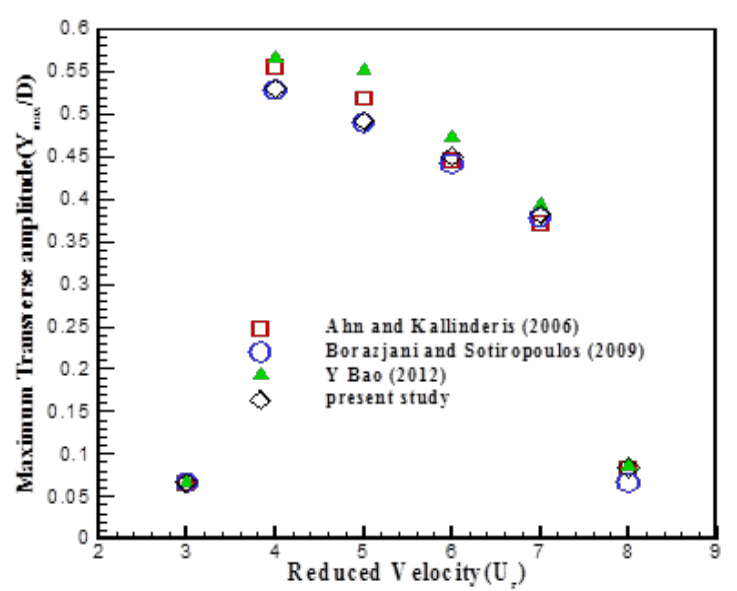

Figure 2 Comparison of maximum Transverse.

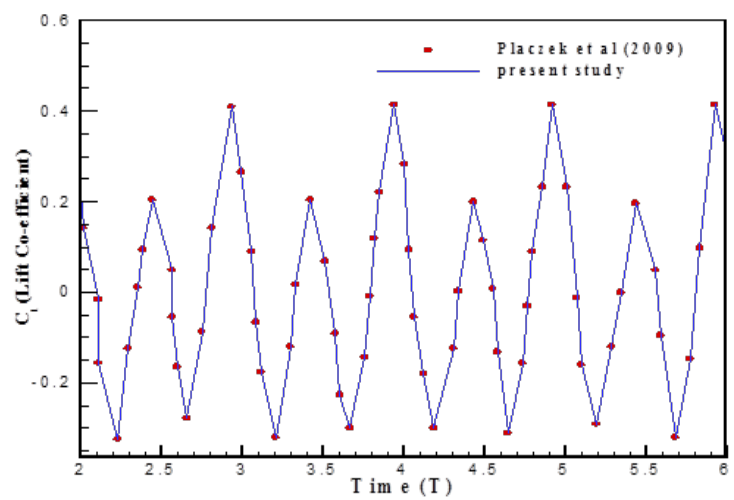

Figure 3 Time series of the fluctuating lift amplitude of single circular cylinder undergoing coefficient at $f_{0}=0.084 \mathrm{VIV}$ with $\mathrm{m}=8 / \pi$ at $\mathrm{Re}=150$.

\section{Conclusions and contribution}

An accurate Harten Lax and van Leer with contact for artificial compressibility (HLLC-AC) Riemann solver with Arbitrarily Lagrangian-Eulerian (ALE) $)^{15}$ formulation has been developed and used for computing flow past an oscillating cylinder. The results obtained by the present solver matches well with that reported in the literature.

\section{Acknowledgments}

None.

\section{Conflicts of interest}

The authors declare that there is no conflicts of interest.

\section{References}

1. Bishop R, Hassan A. The lift and drag forces on a circular cylinder oscillating in a flowing fluid. Proceedings of the Royal Society of London A: Mathematical, Physical and Engineering Sciences. 1964;277(1368):51-75.

2. Griffin OM, Ramberg SE. The vortex-street wakes of vibrating cylinders. Journal of Fluid Mechanics. 1974;66(3):553-576.

3. Khalak A, Williamson C. Dynamics of a hydro elastic cylinder with very low mass and damping. Journal of Fluids and Structures. 1996;10(5):455-472.

Citation: Sonawane CR, More WB. High order accurate numerical simulation of flow past an oscillating circular cylinder. Fluid Mech Res Int J.

2018;2(5):230-232. DOI: I0.15406/fmrij.2018.02.00042 
4. Mittal S, Kumar V. Finite element study of vortex-induced crossflow and in-line oscillations of a circular cylinder at low Reynolds numbers. International Journal for Numerical Methods in Fluids. 1999;31(7):1087-1120.

5. Zhou C, So R, Lam K. Vortex-induced vibrations of an elastic circular cylinder. Journal of Fluids and Structures. 1999;13(2): 165-189.

6. Williamson C, Govardhan G. Vortex-induced vibrations. Annual Review of Fluid Mechanics. 2004;36:413-455.

7. Ahn HT, Kallinderis Y. Strongly coupled flow/structure interactions with a geometrically conservative ALE scheme on general hybrid meshes. Journal of Computational Physics. 2006;219(2):671-696.

8. Borazjani I, Sotiropoulos F. Vortex-induced vibrations of two cylinders in tandem arrangement in the proximity-wake interference region. Journal of Fluid Mechanics. 2009;621:321-364.

9. Raghavan K, Bernitsas M. Experimental investigation of Reynolds number effect on vortex induced vibration of rigid circular cylinder on elastic supports. Ocean Engineering. 2011;38(5):719-731.

10. Bao Y, Huang Cheng, Zhou Dai, et al. Two-degree-of-freedom flowinduced vibrations on isolated and tandem cylinders with varying natural frequency ratios. Journal of Fluids and Structures. 2012;35:50-75.

11. Placzek A, Sigrist JF, Hamdouni A. Numerical simulation of an oscillating cylinder in a cross-flow at low Reynolds number: Forced and free oscillations. Computers \& Fluids. 2009;38(1):80-100.
12. Mandal JC, Sonawane CR, Iyer AS, et al. Incompressible flow computations over moving boundary using a novel upwind method. Computers \& Fluids. 2011;46(1):348-352.

13. Sonawane CR, Mandal JC. Numerical simulation of flow inside differentially heated rotating cavity. International Journal of Numerical Methods for Heat and Fluid Flow. 2013;23(1):23-54.

14. Sonawane CR, Mandal JC. Simulation of moderator flow and temperature inside calandria of CANDU reactor using artificial compressibility method. Journal of Heat Transfer Engineering. 2014;35(11):1254-1266.

15. Cook JL, Hirt CW, Amsden AA. An arbitrary Lagrangian-Eulerian computing method for all flow speeds. Journal of Computational Physics. 1974;14(3):227-253.

16. Bijl H, de Boer A, van der Schoot MS. Mesh deformation based on radial basis function interpolation. Computers and Structures. 2007;85(11):784 795.

17. Sonawane CR, Mandal JC, Roa SP. High-Resolution Incompressible Flow Computations over Unstructured Mesh using SDWLS Gradients. Journal of The Institution of Engineers(India):Series C. 2017:1-14.

18. Chorin AJ. Numerical solution of Navier-Stokes equations. Math Comput. 1968;22:745-762.

19. Lombard CK, Thomas PD. Geometric conservation law and its application to flow computations on moving grids. AIAA Journal. 1979;17:1030-1037. 